\title{
FACTORES ECONÓMICOS EXTERNOS EN EL CRECIMIENTO DE LA ECONOMÍA PERUANA: UN MODELO VECTOR AUTORREGRESIVO (VAR)
}

\author{
EXTERNAL ECONOMIC FACTORS IN THE GROWTH OF THE PERUVIAN ECONOMY: \\ AN AUTOREGRESSIVE VECTOR MODEL (VAR)
}

Iraida Betty Chavez Lazo Universidad Nacional Mayor de San Marcos Lima, Perú

ORCID: https://orcid.org/0000-0003-1797-1455 Correo electrónico: iraida.chavez@unmsm.edu.pe

Víctor Fernando Jesús Burgos Zavaleta Universidad Nacional Mayor de San Marcos Lima, Perú ORCID: https://orcid.org/0000-0001-7530-2669 Autor para correspondencia: vburgosz@unmsm.edu.pe

\section{RESUMEN}

Objetivo: Determinar el efecto que generan los factores económicos externos hacia el crecimiento económico del Perú en el periodo 2000-2019. Método: La investigación fue de índole descriptivo y explicativo, con diseño no experimental. La técnica de recolección de data empleó el análisis documentario y utilizó una población de tipo censal, considerando a la economía del Perú y la economía global. Se estimó el modelo vector autorregresivo (VAR), donde se desarrolló un análisis estadístico y un análisis estructural. Resultados: Se encontró que el shock del producto bruto interno (PBI) chino da a conocer un crecimiento en su participación $(42,0 \%)$ sobre el crecimiento del PBI del Perú en su décimo trimestre; los shocks de los términos de intercambio registraron una incidencia económica que oscila alrededor del 2,0\% y los shocks del índice de riesgo país han consignado un leve ascenso, alcanzando el 3,0\% de variabilidad del PBI del Perú. Conclusión: Se encontró que las variables consideradas como factores externos, sobre todo el $\mathrm{PBI}$ de China, repercuten, de una manera u otra, en el crecimiento económico del país.

Palabras clave: Factores económicos externos; producto bruto interno; vector autorregresivo.

\begin{abstract}
Objective: Determine the effect of external economic factors on Peru's economic growth in the period 2000-2019. Method: The investigation has a descriptive and explanatory nature, with a non-experimental design. The data collection technique employed the documentary analysis and used a census type population, considering the Peruvian and global economy. The Vector Autoregressive (VAR) model was estimated, from which a statistical and a structural analysis were developed. Results: It was found that the shock of the Chinese gross domestic product (GDP) reveals a growth in its participation $(42,0 \%)$ on the growth of GDP in Peru in its 10 th quarter. The shocks of the exchange terms registered an economic impact that fluctuates around 2,0\% and the shocks of the country risk index have registered a slight increase, reaching $3,0 \%$ of the GDP variability of Peru. Conclusion: It was found that the variables considered as external factors, above all the China's GDP, have repercussions, in one way or another, on the economic growth of the country.
\end{abstract}

Keywords: External economic factors; gross domestic product; vector autoregressive.

(c) Los autores. Este artículo es publicado por la revista Quipukamayoc de la Facultad de Ciencias Contables, Universidad Nacional Mayor de San Marcos. Este es un artículo de acceso abierto, distribuido bajo los términos de la licencia Creative Commons Atribución 4.0 Internacional (CC BY 4.0) [https://creativecommons.org/licenses/ by/4.0/deed.es] que permite el uso, distribución y reproducción en cualquier medio, siempre que la obra original sea debidamente citada de su fuente original. 


\section{INTRODUCCIÓN}

En la literatura, se ha encontrado diversa información relativa a los efectos de los factores económicos externos en las economías abiertas. Desde de tal perspectiva, el Perú, a lo largo de su historia, al ser una economía dependiente del comercio internacional, ha tenido un comportamiento económico producto de su exposición a los shocks económicos externos.

En efecto, el Financial Times Stock Exchange (2017) clasifica como economías emergentes a las de Chile, Colombia y Perú. Sobre ello, Peñaranda (2016) muestra la debilidad de tales economías, integrantes de la Alianza del Pacífico, frente a los impactos del entorno internacional y cómo esto ha influido en la merma de la inversión de capital. Recientemente, Castillo (2021) muestra en su investigación esta fuerte interdependencia entre los factores de decisión de inversión directa y los mercados financieros de las economías mencionadas.

Por su parte, Jurado y Ramos (2021) resaltan la importancia de evaluar la repercusión de los factores económicos externos sobre el crecimiento económico del Perú, indicando los efectos positivos que generan el PBI de China y Estados Unidos, así como los términos de intercambio, mientras que la tasa de política monetaria del Sistema de Reserva Federal repercutió negativamente en la economía nacional. Por otro lado, Quispe, Rodríguez, Toma y Vásquez (2017), en su investigación sobre la dependencia recíproca entre los mercados de valores americanos, se centraron en detectar las posibles vinculaciones de interdependencia como síntoma de vulnerabilidad al contagio financiero.

En ese contexto, Nolazco, Legua-Lafosse y Céspedes (2016) señalan que la economía peruana, al ser pequeña y abierta, es más propensa a ser afectada por los shocks externos, debido a que tiene menor capacidad para hacer frente a los acontecimientos económicos desencadenados en las grandes economías. Además, en el estudio realizado por Rodríguez y Díaz (2020), se identificó que los países de América Latina son fácilmente influenciados ante posibles shocks de crecimiento por parte de China y Estados Unidos, por medio de tres canales: el comercio, los precios de las materias primas y las corrientes financieras.

$\mathrm{Al}$ respecto, para el presente estudio se seleccionaron tres variables en los canales de transmisión de los shocks externos, los cuales son los siguientes: producto bruto interno de China, término de intercambio y riesgo país.

El criterio utilizado para la selección del producto bruto interno de China como variable se debió a que China es el principal socio comercial del Perú y, de acuerdo con la Figura 1, registra un $27 \%$ de participación en el comercio nacional, superando en más de 10 puntos porcentuales (p.p.) a Estados Unidos y en 6 p.p. a los países de América Latina en su conjunto, destacando las exportaciones mineras tradicionales. Esto demuestra la dependencia comercial que tiene Perú de China y la envergadura económica que causa algún impacto sobre dicha variable.

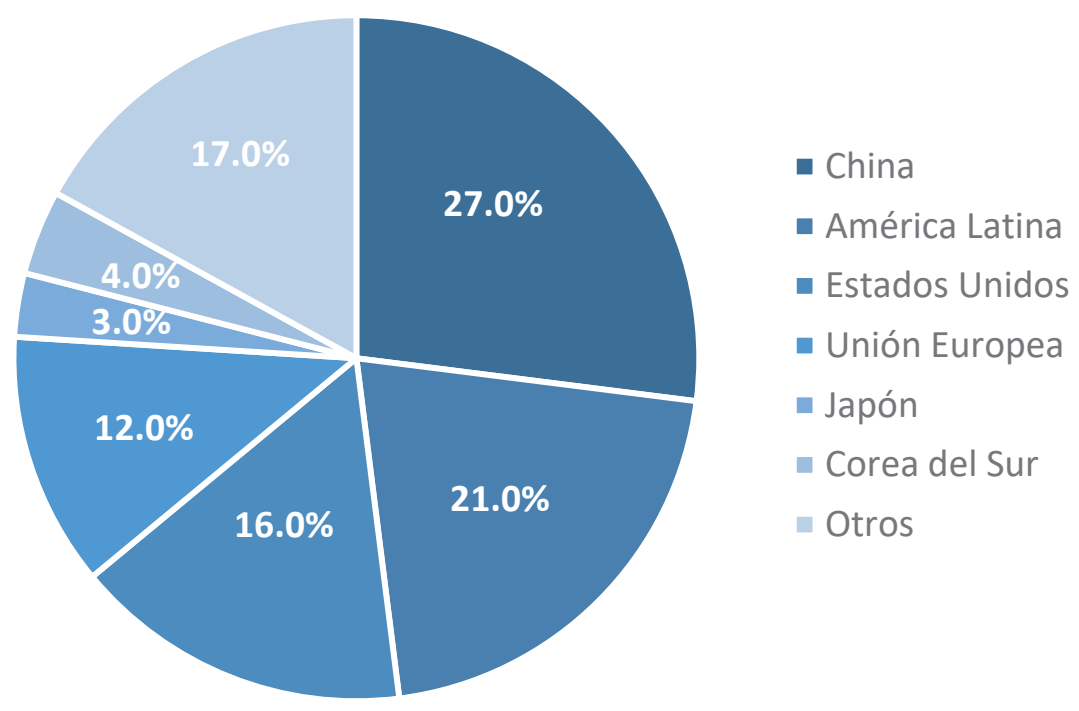

Figura 1. Principales socios comerciales del Perú, 2019 (Expresado en porcentaje)

Fuente: Elaboración propia sobre la base de los datos del Banco Central de Reserva del Perú (2019a) 
Asimismo, Nolazco et al. (2016) hacen referencia al canal de transmisión comercial, en el cual se aprecia el flujo económico que se da en la economía del Perú a partir de la variación del producto bruto interno de China. En ese sentido, indican que dicho canal "se activa con cambios en el crecimiento de los principales socios comerciales” (p. 9), entre los que destaca China, llegando a ser el $21 \%$ del total de las exportaciones en el 2014. Dichas exportaciones están compuestas principalmente de productos tradicionales. Del mismo modo, sobre los canales de transmisión, se afirma lo siguiente:

Los principales canales, por los cuales el crecimiento económico de China afecta a Perú, son: (i) directamente, a través del comercio y la inversión extranjera directa de China; (ii) indirectamente, mediante variaciones de los precios de las materias primas. (Banco Mundial, 2016, como se citó en Nolazco, et al., 2016, p. 12)

Adicionalmente, de acuerdo con el modelo teórico Mundell-Fleming, en el caso de una economía abierta, se señala que la relación de la producción externa es positiva, puesto que se produce un impacto positivo a través de las exportaciones netas.

Por otro lado, la variable de términos de intercambio es seleccionada como factor económico externo, puesto que el Perú, al ser un país económicamente pequeño, es vulnerable a las variaciones en los precios tanto de sus exportaciones como importaciones y estos, a su vez, repercuten en los términos de intercambio.

De acuerdo con el Banco Central de Reserva del Perú (2019b), los términos de intercambio en el 2019, por el lado de los precios de exportaciones, tuvieron una caída de $-1,8 \%$, en virtud del desplome de los precios de los commodities (excepto el precio del oro que se ha fortalecido) y, por el lado de los precios de importaciones, se ha observado un incremento en el precio del maíz.

La investigación de la Comisión Económica para América Latina y el Caribe (2018) señala la preponderancia de las variables externas, como los términos de intercambio, sobre el crecimiento económico de las economías emergentes; adicionalmente, precisa lo siguiente:

Para economías altamente abiertas como las de América Latina y el Caribe, el desempeño de la inversión y el crecimiento se vuelve altamente dependiente de las condiciones externas. Esto se da particularmente en aquellas economías cuya estructura productiva y la composición de la inversión están muy concentradas en sectores expuestos a los vaivenes de la economía internacional. (p. 197)
Este sería claramente el caso de la economía peruana, la cual es exportadora, principalmente, de productos tradicionales e importadora de insumos para su industria.

En relación con el canal de transmisión de los términos de intercambio, Nolazco et al. (2016) mencionan que "se activa cuanto los precios de los productos de exportación se modifican significativamente" (p. 2). Asimismo, señalan que las economías de los principales socios comerciales del Perú repercuten en los precios de exportación, destacando el precio de las materias primas, tales como, los metales y el petróleo.

Respecto de la variable riesgo país, se consideró como el diferencial entre la tasa de interés de bonos provenientes de Estados Unidos y los bonos del Perú. Se toma como factor económico externo, ya que, en el campo específico de los mercados bursátiles, Quispe et al. (2017) y Ticona (2019) identificaron la influencia de los choques financieros bursátiles externos en la rentabilidad de las empresas colombianas y las empresas peruanas, respectivamente. Adicionalmente, Peña (2017) encontró que el riesgo país repercute en la inversión extranjera directa y esta, a su vez, tiene implicancia sobre el crecimiento económico del Perú, dado que es una economía abierta y tiene alto grado de dependencia internacional.

Ante ello, radica la importancia de analizar el comportamiento de estos factores económicos externos sobre el crecimiento del producto bruto interno del Perú, toda vez que las entidades pertinentes puedan evaluar las políticas económicas adecuadas para hacer frente a la influencia recibida ante las condiciones externas ya mencionadas.

Sobre la base de lo antes expuesto, el problema de la presente investigación se enfoca en conocer lo siguiente: ¿Cuál fue la implicancia que tuvieron los factores económicos externos en el crecimiento económico del Perú durante el periodo 2000-2019?, considerando que es una economía emergente y abierta al comercio exterior. A partir de lo cual, la hipótesis planteada es: "Los factores económicos externos repercutieron considerablemente sobre el crecimiento económico del Perú durante el periodo 2000-2019".

\section{MATERIAL Y MÉTODOS}

La investigación propuesta fue de índole descriptivo y explicativo, donde inicialmente se realizó un análisis de la información recabada a fin de caracterizar los diferentes fenómenos económicos externos que influyeron en el crecimiento económico del Perú. Se examinó el comportamiento económico del país frente a efectos del PBI chino, los términos de intercambio y el índice de riesgo 
país. Al respecto, cabe mencionar que dichas variables han sido consideradas en variaciones porcentuales.

El diseño de investigación aplicado para el presente trabajo fue no experimental, puesto que permitió observar los fenómenos económicos ocurridos durante el periodo de estudio 2000-2019 y analizar su repercusión en el crecimiento económico del Perú. Seguidamente, se seleccionó el método longitudinal de tendencia, el cual evaluó las variaciones de las variables durante el tiempo. Además, se escogió una población tipo censal, donde se contempló a la economía peruana y global, tomando países tales como China, al ser uno de los principales socios comerciales del Perú.

Para la recolección de datos confiables, se adoptó la técnica de exploración documental, donde se consideró a las instituciones formales, ya sean nacionales e internacionales, que proporcionaron datos confiables. Dicho diagnóstico se efectuó por medio de la búsqueda de datos, cuya fuente de información secundaria fue recopilada por las instituciones formales estatales peruanas, tales como el Banco Central de Reserva del Perú (BCRP) e Instituto Nacional de Estadística e Informática (INEI), así como instituciones internacionales como el Fondo Monetario Internacional (FMI) y el Banco Mundial (BM), que fueron complementarias a la selección de datos, para cumplir los objetivos de esta investigación.

A fin de poder identificar los efectos o shocks de los factores económicos externos, se aplicó un modelo econométrico de ecuaciones simultáneas llamado modelo vector autorregresivo (VAR), el cual está constituido por un sistema de ecuaciones en su forma reducida. Mediante la siguiente ecuación, se expresó el modelo VAR en su forma reducida:

$$
\begin{gathered}
y_{t}=A_{0}+A_{1} y_{t-1}+u_{t} \\
{\left[\begin{array}{c}
p b i_{t} \\
p b i_{c h i_{t}} \\
t i_{t} \\
e m b i_{t}
\end{array}\right]=\left[\begin{array}{l}
a_{10} \\
a_{20} \\
a_{30} \\
a_{40}
\end{array}\right]+\left[\begin{array}{llll}
a_{11} & a_{12} & a_{13} & a_{14} \\
a_{21} & a_{22} & a_{23} & a_{24} \\
a_{31} & a_{32} & a_{33} & a_{34} \\
a_{41} & a_{42} & a_{43} & a_{44}
\end{array}\right]\left[\begin{array}{c}
p b i_{t-1} \\
p b i_{c h i_{t-1}} \\
t i_{t-1} \\
e m b i_{t-1}
\end{array}\right]+\left[\begin{array}{c}
e_{p b i_{t}} \\
e_{p b i_{c h i t}} \\
e_{t i_{t}} \\
e_{e m b i_{t}}
\end{array}\right]}
\end{gathered}
$$

Donde " $y_{t}$ " es un vector de variables principales, " $y_{t-1}$ " es un vector de rezago, $y$ " $u_{t}$ " es una matriz de identificación de los factores externos.

Siendo que el:

$p b i_{t}=$ Producto bruto interno del Perú

$p b i_{c h i_{t}}=$ Producto bruto interno de China

$t i_{t}=$ Términos de intercambio

embi $i_{t}$ Índice de riesgo país
Luego de estimar el modelo, se procedió a hacer un análisis estructural, compuesto por una evaluación de las simulaciones de impulso-respuesta, la descomposición de varianza del error de predicción y la descomposición histórica.

Además, se realizó un análisis estadístico de la variable dependiente, en este caso, la variación anual histórica del producto bruto interno del Perú, a fin de poder evaluar su comportamiento en el periodo de estudio, 2000-2019.

Un hecho importante en los modelos VAR son los choques tecnológicos o innovaciones (conocido como término de error en modelos de corte transversal, pero no en series de tiempo) que, al ser modelos dinámicos, agregan a todas las variables omitidas y son modeladas en las ecuaciones, cuyos impactos pueden ser evaluados a través de las funciones impulso-respuesta. Esto, a su vez, permite centrar o modelar únicamente las variables más relevantes, según los objetivos del estudio, sin afectar o alterar el choque puro de las variables del modelo.

\section{RESULTADOS}

El producto bruto interno peruano ha registrado cambios cíclicos contractivos y expansivos en el periodo 2000 2019. Estas alteraciones se vieron influenciadas por el comportamiento de distintos indicadores macroeconómicos, sobre todo en el comercio, tomando mayor preponderancia para el Perú en los últimos años. De acuerdo con la información presentada en la Figura 2, la economía nacional ha estado sujeta a cambios en las últimas décadas, producto de acontecimientos externos que repercutieron en su crecimiento económico.

A inicios del año 2000, se logró una variación anual de $2,7 \%$. Posterior a ello, en el 2001, se registró una caída en la tasa de crecimiento en 2,1 p.p. a causa de inestabilidades políticas que repercutían en el índice de riesgo país. Seguidamente, desde el año 2002 a 2008, se recoge una tendencia creciente que en promedio fue de 6,6 p.p.

En el año 2009, se experimentó un descenso a 1,0\% en su tasa de crecimiento anual, debido a la crisis financiera internacional originada en Estados Unidos en el 2008. A partir del año 2010 al 2014, la variación anual fue decreciente, pero positiva (de 5,8 p.p. en promedio), mientras que, en el año 2019, la tasa de crecimiento fue de $2,6 \%$, respecto del año anterior.

Según el Banco Central de Reserva del Perú (2017), la crisis internacional generó una caída de términos de intercambio de 13,0\%. Desde el 2003 al 2008, se observa un crecimiento del PBI nacional que, en el 2008, tuvo una variación del 9,1\%. Posterior a ello, en el 2009, el Perú registró una evidente desaceleración con un crecimiento de la actividad 


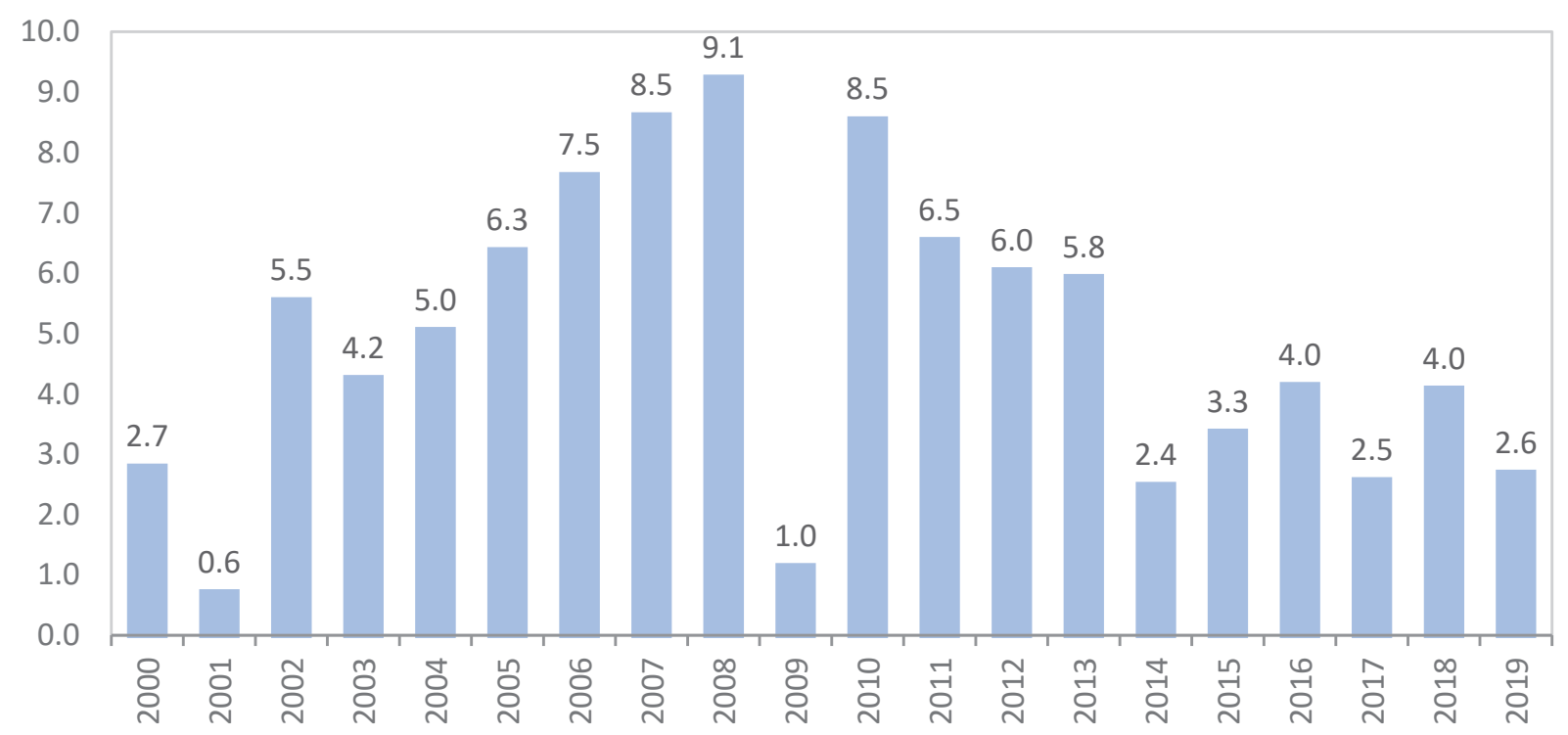

Figura 2. Variación del producto bruto interno del Perú, 2000-2019 (Expresado en porcentaje)

Fuente: Elaboración propia sobre la base de los datos del Fondo Monetario Internacional (2019)

económica únicamente del $1,0 \%$ como inferencia de los efectos del desequilibrio financiero internacional.

Asimismo, según información obtenida del Instituto Nacional de Estadística e Informática (2020), el PBI del Perú tuvo una variación anual de 2,2\%, uno de los más bajos de la última década, y una caída de 1,8 p.p. respecto del año anterior, a causa del descenso de la producción pesquera y minera.

\section{Análisis estructural}

En esta sección, se presentará los resultados empíricos producto de la estimación del modelo vector autorregresivo (VAR). Este modelo permitió analizar el impacto de los factores económicos externos, tales como el producto bruto interno de China, el índice de riesgo país y los términos de intercambio sobre el crecimiento económico del Perú. En ese sentido, se desarrolló el diagnóstico mediante el análisis impulso-respuesta, la descomposición de varianza del error de predicción y la descomposición histórica, cuyos resultados se presentan en la Figura 3.

\section{Análisis impulso-respuesta}

a. Respuesta del crecimiento económico del Perú frente a un shock de una desviación estándar en el crecimiento del PBI de China (PBI_China):

Se revisó la respuesta del crecimiento del PBI del Perú, en un horizonte de 10 trimestres ante un shock de una desviación estándar en el crecimiento del producto de China. Como se puede apreciar, a partir del primer hasta el tercer trimestre tiene un impacto positivo a una tasa de $0,1 \%$ en promedio. Posteriormente, presenta una caída hasta el décimo periodo, alcanzando su pico más bajo en el octavo trimestre con un impacto negativo de 1,0 p.p.

b. Respuesta del crecimiento económico del Perú frente a un shock de una desviación estándar en la variación del riesgo país (EMBIGALSP):

Se evaluó la reacción del crecimiento del PBI del Perú, en un horizonte de 10 trimestres ante un shock de una desviación estándar en la variación del riesgo país de América Latina sin Perú. Como se puede observar los dos primeros trimestres aportan impactos negativos de 0,3 p.p. Luego, en los siguientes trimestres hasta el décimo, proporciona impactos positivos crecientes hasta lograr un impacto de 5,1 p.p.

c. Respuesta del crecimiento económico frente a un shock de una desviación estándar en la variación de los términos de intercambio (TI):

Se analizó la respuesta del crecimiento del PBI nacional, en un horizonte de 10 trimestres, ante un shock de una desviación estándar en la variación de los términos de intercambio. Como se puede observar, desde el primer trimestre surge un impacto positivo en 


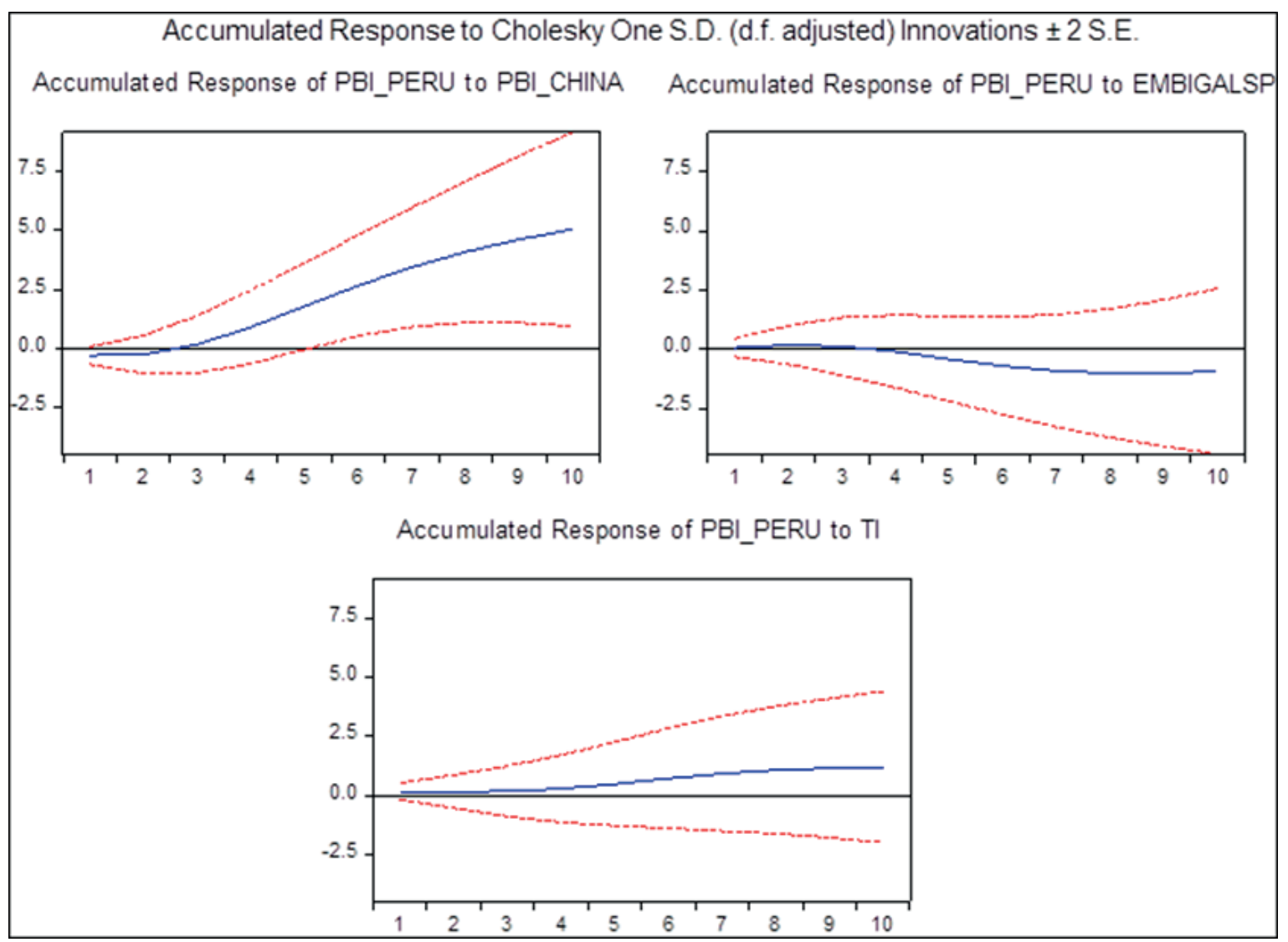

Figura 3. Funciones de impulso-respuesta del crecimiento económico del Perú ante factores económicos externos Fuente: Elaboración propia sobre la base de los datos del Fondo Monetario Internacional (2019)

crecimiento, llegando a su punto máximo en el último periodo de estudio (décimo trimestre), registrando un impacto de 1,2 p.p.

\section{Descomposición de varianza del error de predicción}

Otro tipo de análisis que se realizó para evaluar la preponderancia de los factores económicos externos sobre el crecimiento de la economía nacional es la descomposición de la varianza, la cual permitió observar la variabilidad del indicador e informar, en diferentes trimestres, el porcentaje de volatilidad que tiene una variable por los shocks de alguna de las otras variables.

Los resultados obtenidos en la Tabla 1 corroboran la información señalada en el análisis de impulso-respuesta, donde, en el horizonte temporal de 10 trimestres, se logra apreciar que el producto bruto interno de China va adquiriendo preponderancia en el crecimiento económico del Perú; por consiguiente, a partir del tercer trimestre, presenta un aumento en su contribución sobre el producto bruto interno nacional, llegando a explicar, en el décimo trimestre, que más del 42,0\% de la variabilidad del PBI del
Perú depende de la variabilidad del crecimiento del PBI de China.

Por otro lado, los términos de intercambio exponen una representatividad fluctuante, logrando su mayor $(2,3 \%)$ y menor $(0,6 \%)$ nivel de variabilidad del PBI nacional, en el noveno y tercer trimestre, respectivamente. Del mismo modo, la variable riesgo país registró una tendencia creciente, logrando un 3,7\% de variabilidad del PBI del Perú en el séptimo trimestre. A partir de dicho periodo fue disminuyendo su participación hasta 3,5\%.

\section{Descomposición histórica}

En la Figura 3, se muestra la descomposición histórica de los factores económicos externos utilizados, entre ellos se encuentran el producto bruto interno de China, el índice de riesgo país y los términos de intercambio. Como se puede observar, el PBI de China tiene la mayor representatividad en el crecimiento del PBI peruano, respecto de las demás variables durante el periodo 2000-2019, alcanzando su mayor participación positiva en el tercer trimestre del 2008 y su caída más significativa en el primer trimestre del 2019. 
Tabla 1

Análisis de descomposición de varianza del crecimiento del PBI del Perú (Expresado en porcentajes y desviaciones estándar)

\begin{tabular}{|c|c|c|c|c|c|}
\hline Periodo & S.E. & PBI_CHINA & $\mathrm{TI}$ & EMBIGALSP & PBI_PERU \\
\hline \multirow{2}{*}{1} & 0,766006 & 3,191462 & 1,137817 & 0,168792 & 95,50193 \\
\hline & & $(3,85284)$ & $(2,48807)$ & $(1,42765)$ & $(4,94940)$ \\
\hline \multirow{2}{*}{2} & 1,190267 & 1,902308 & 0,669355 & 0,376027 & 97,05231 \\
\hline & & $(3,31566)$ & $(2,29320)$ & $(2,88693)$ & $(5,36780)$ \\
\hline \multirow{2}{*}{3} & 1,515971 & 5,440025 & 0,586980 & 0,380757 & 93,59224 \\
\hline & & $(5,97090)$ & $(2,73390)$ & $(4,00741)$ & $(8,06611)$ \\
\hline \multirow{2}{*}{4} & 1,750504 & 14,68119 & 0,746969 & 1,261678 & 83,31017 \\
\hline & & $(9,35127)$ & $(2,86188)$ & $(4,25735)$ & $(10,6123)$ \\
\hline \multirow{2}{*}{5} & 1,910918 & 24,46801 & 1,278686 & 2,590344 & 71,66296 \\
\hline & & $(11,0623)$ & $(3,22434)$ & $(4,49553)$ & $(11,6964)$ \\
\hline \multirow{2}{*}{6} & 2,018914 & 31,54118 & 1,845639 & 3,432241 & 63,18094 \\
\hline & & $(11,7626)$ & $(3,89907)$ & $(4,93960)$ & $(12,1140)$ \\
\hline \multirow{2}{*}{7} & 2,093301 & 36,15538 & 2,195741 & 3,665905 & 57,98297 \\
\hline & & $(12,1828)$ & $(4,44886)$ & $(5,32183)$ & $(12,3350)$ \\
\hline \multirow{2}{*}{8} & 2,146843 & 39,12869 & 2,325259 & 3,583590 & 54,96246 \\
\hline & & $(12,4879)$ & $(4,81139)$ & $(5,62068)$ & $(12,4904)$ \\
\hline \multirow{2}{*}{9} & 2,186990 & 41,02745 & 2,331735 & 3,466928 & 53,17389 \\
\hline & & $(12,7186)$ & $(5,07183)$ & $(5,96382)$ & $(12,6322)$ \\
\hline \multirow{2}{*}{10} & 2,217854 & 42,23243 & 2,298460 & 3,451863 & 52,01725 \\
\hline & & $(12,9073)$ & $(5,24576)$ & $(6,37230)$ & $(12,7493)$ \\
\hline
\end{tabular}

Fuente: Elaboración propia sobre la base de los datos del Fondo Monetario Internacional (2019)

Todo ello debido a las tensiones comerciales con Estados Unidos y una caída del consumo interno.

Posteriormente, los términos de intercambio, como segunda variable, presenta mayor repercusión en el crecimiento PBI nacional. Dicha variable muestra oscilaciones constantes en los precios de los minerales, el petróleo, el maíz y el trigo. De acuerdo con el Banco Central de Reserva del Perú (2019a), las principales causas de las variaciones respecto de los precios en los commodities se deben, principalmente, a "la guerra comercial entre Estados Unidos y China, así como a la incertidumbre que generó el Brexit y los riesgos geopolíticos en Medio Oriente" (p. 53). Así también, se identificó que el riesgo país y los demás factores impactan en menor proporción al producto bruto interno del Perú. Cabe mencionar que, en la Figura 4, se ha contemplado la variación porcentual del PBI nacional.

En otro punto de análisis, a fin de poder obtener un mayor detalle sobre la implicancia de cada variable económica externa considerada en el presente estudio, se elaboró la Figura 5, según los datos obtenidos en el tercer trimestre del 2019. En dicha figura, se observa la barra denominada
“PBI Perú sin CE” (condiciones externas), asumiendo que, en caso no hubieran existido las condiciones externas, el crecimiento del PBI peruano hubiera registrado 4,9 p.p.

Se logra apreciar que los impactos del PBI de China (-1,6 p.p.) y del riesgo país (-0,4 p.p.) sobre el crecimiento del producto del Perú son mayores que la repercusión de los términos de intercambio ( 0,1 p.p.). Asimismo, existieron otros factores que no son objeto del presente análisis y que, en este caso, tienen un impacto positivo de 0,2 p.p. Finalmente, da como resultado que el crecimiento del PBI nacional con contribución externa fue de 3,3 p.p.

\section{DISCUSIÓN}

Los resultados obtenidos permitieron comprobar la hipótesis planteada referente a la repercusión de los factores económicos externos en el crecimiento económico del Perú. En tanto, esto permitió cuantificar su efecto o shock y evaluar la temporalidad de los tres shocks analizados: los términos de intercambio, producto bruto interno China y el riesgo país. 


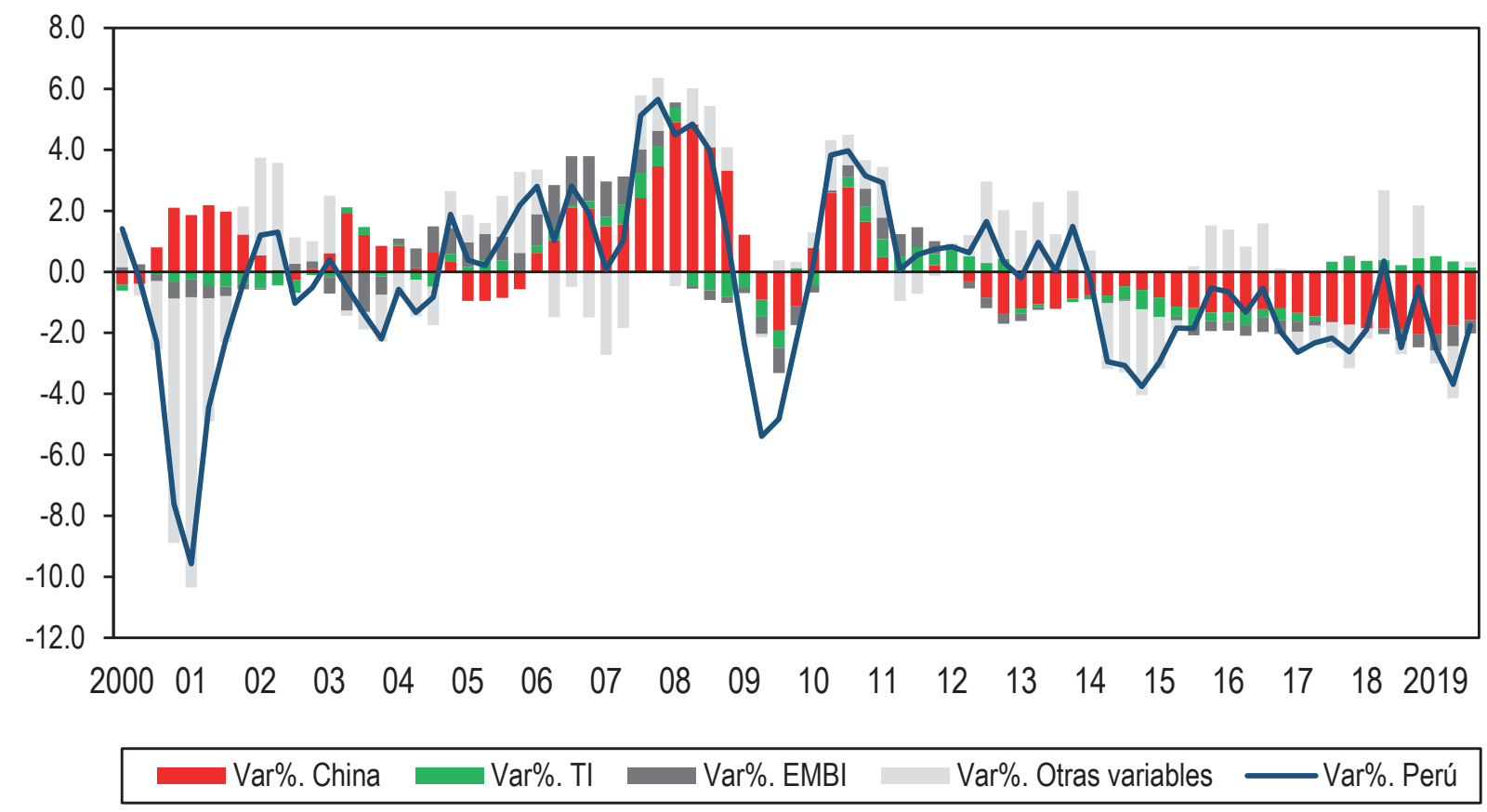

Figura 4. Descomposición historia del crecimiento del PBI del Perú 2000-2019 (Expresado en porcentaje)

Fuente: Elaboración propia sobre la base de los datos del Fondo Monetario Internacional (2019) y el Banco Central de Reserva del Perú (2019a)

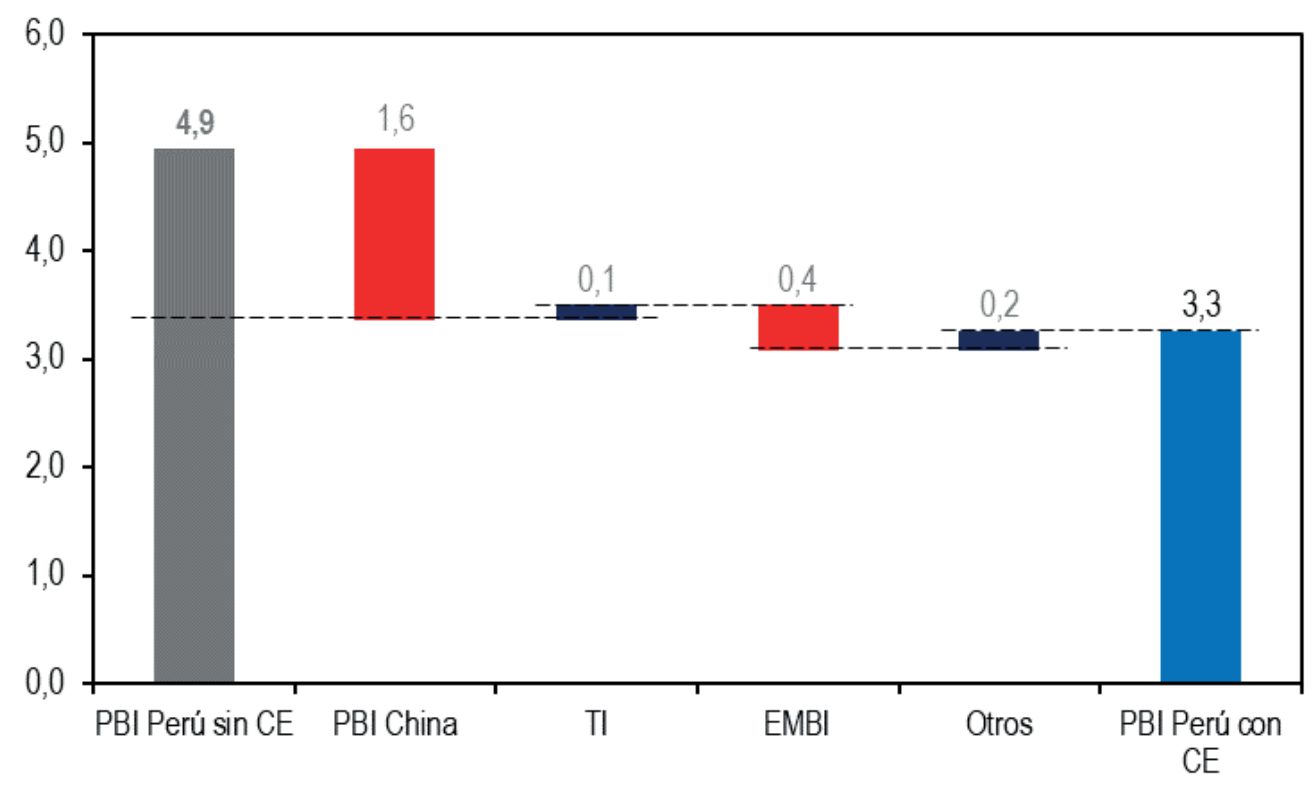

Figura 5. Contribuciones en el crecimiento del PBI del Perú (Expresado en porcentaje)

Fuente: Elaboración propia sobre la base de los datos del Fondo Monetario Internacional (2019) y el Banco Central de Reserva del Perú (2019a) 
Relativo a los shocks del producto bruto interno de China analizados en un horizonte de tiempo de diez trimestres, se observó que, en el primer periodo de estudio, el PBI de China solo contaba con una participación de alrededor del 3,0\% de variabilidad del crecimiento del PBI peruano. Posteriormente, a partir del tercer trimestre, se experimentó un crecimiento constante e ininterrumpido hasta el décimo periodo, logrando alcanzar en este último el 42,0\% de variabilidad del PBI nacional.

Estos resultados responden a que, al ser China el principal socio comercial del Perú, todo impacto en su PBI repercute económicamente en el crecimiento económico nacional, dado que la demanda de materias primas, especialmente del sector minero, se ve involucrada.

En ese sentido, los resultados de Jurado y Ramos (2021) reafirman la tendencia del presente estudio, precisando que el PBI de China, al ser uno los principales socios comerciales desde el 2011, ha provocado una apreciación transitoria en el crecimiento del PBI peruano, en tanto, infieren lo siguiente:

Una innovación del PBI China es significativa para nuestra variable, esto indica que un incremento del mismo generará una reacción positiva en la variable domestica [sic] PBI, lo cual se muestra claramente en el tercer periodo del modelo, donde el PBI alcanza un máximo incremento. (p. 51)

Se muestra entonces, mediante la evidencia de este estudio, la fuerte correlación positiva entre el crecimiento de la economía en China y Perú.

Por otro lado, los shocks de los términos de intercambio registraron una participación en el crecimiento económico del Perú, que oscila alrededor del 2,0\%, a lo largo de los 10 primeros periodos, alcanzando su menor participación en el tercer periodo $(0,6 \%)$, mientras que su mayor participación se dio en el octavo periodo (2,3\%) sobre la variabilidad del PBI nacional.

Este estudio afianzó la idea planteada, donde los shocks de los términos de intercambio repercuten positivamente en la economía del Perú. Por medio del documento anual elaborado por la Comisión Económica para América Latina y el Caribe (2018), se identificó cierto grado de heterogeneidad en las economías emergentes, dado que "las proyecciones correspondientes a Bolivia, Chile, Colombia, Paraguay y Perú se han revisado al alza (principalmente por la mejora de sus términos de intercambio), mientras que en otros casos las previsiones de crecimiento se mantienen o disminuyen levemente" (p. 100), afianzando, de esta manera, la importancia de los términos de intercambio en las fluctuaciones del PBI peruano.
Los shocks del riesgo país han registrado un leve crecimiento durante los 10 primeros trimestres, obteniendo en el último periodo el 3,0\% de variabilidad del crecimiento del PBI peruano. Se puede apreciar que va adquiriendo mayor preponderancia, producto de acontecimientos políticos o decisiones en materia económica que, de una u otra forma, generan cierta inestabilidad en el país, lo cual repercute en mayor cuantía en la reputación crediticia del país.

Referente a lo antes mencionado, cabe precisar que el Perú, del mismo modo que sus pares latinos, ha descubierto históricamente un comportamiento volátil en materia económica, social y financiera, puesto que ha experimentado etapas de crisis económicas, financieras, políticas y sociales, y ello ha repercutido en las empresas extranjeras y nacionales. En esa línea, Peña (2017) colige que "actualmente se considera que Perú es una economía confiable ya que tiene un riesgo país relativamente bajo, por lo que resulta (en teoría) atractivo invertir en el país" (p. 40). Téngase en cuenta que el riesgo país del Perú, que durante el 2017 se mantuvo en promedio en 145 puntos EMBIG, permanece relativamente estable hasta hoy, alcanzado, durante el primer semestre del 2021, los 155 puntos EMBIG. Asimismo, obsérvese que la tasa de interés a 10 años de los bonos peruanos en USD, que durante el 2017 alcanzó un promedio de $3,13 \%$, durante lo que va del 2021, ha mostrado un promedio de $2,55 \%$, reflejando una disminución del costo del capital para el Gobierno peruano producto del incremento de la confianza de los inversionistas.

Luego del análisis realizado, se concluye que, efectivamente, los factores económicos externos repercuten notablemente en el crecimiento económico del Perú, sobre todo el efecto o shock del producto bruto interno de China, al ser el principal socio comercial de materias primas mineras, adquiriendo mayor preponderancia sobre la economía del país.

Adicionalmente, dado el entorno inesperado suscitado producto de la pandemia del COVID-19, las economías a nivel mundial han sufrido una recesión. Esto ha desencadenado severos problemas económicos sobre las economías emergentes como es el caso del Perú, debido a que, adicionalmente a los problemas económicos internos presentados como producto de la crisis sanitaria a nivel mundial, estas han sido vulnerables a efectos adversos externos, debido a la caída en el intercambio comercial.

En ese sentido, resulta importante proponer objetivos claros de política económica, puesto que, al ser una economía emergente y abierta, seguirá estando expuesta a posibles fluctuaciones en el ámbito internacional. Por ello, se debe considerar la implementación de políticas que permitan aminorar los efectos económicos externos perjudiciales a 
la economía del Perú, contemplando también la crisis económica producto de la pandemia del COVID-19.

Finalmente, luego de lo antes visto, se plantea lo siguiente: ¿Serán más marcados los impactos de los factores económicos externos a consecuencia de la pandemia del COVID-19?; ¿aportarían la diversificación de las exportaciones y el impulso de las exportaciones no tradicionales a disminuir el efecto de los factores externos en la variabilidad de la economía peruana? y ¿aumentará o disminuirá en los años siguientes el impacto de las variaciones de la economía china sobre la economía peruana? Estas y otras interrogantes podrán marcar rutas de estudio a futuros investigadores que tengan interés en continuar y profundizar la presente investigación.

\section{REFERENCIAS}

Banco Central de Reserva del Perú. (2017). Memoria 2017. Recuperado de https://www.bcrp.gob.pe/publicaciones/memoria-anual/memoria-2017.html

Banco Central de Reserva del Perú. (2019a). Memoria 2019. Recuperado de https://www.bcrp.gob.pe/publicaciones/memoria-anual/memoria-2019.html

Banco Central de Reserva del Perú. (2019b). Reporte de Inflación. Diciembre 2019. Panorama actual y proyecciones macroeconómicas 2019-2021. Recuperado de https://www.bcrp.gob.pe/publicaciones/reporte-de-inflacion.html

Castillo, J. (2021). Factores determinantes en las decisiones de inversión y desarrollo de los mercados de países emergentes, periodo 2011-2015 (Tesis para optar el grado de magíster en Economía con mención en Finanzas). Universidad Nacional Mayor de San Marcos, Lima.

Comisión Económica para América Latina y el Caribe. (2018). Estudio Económico de América Latina y el Caribe 2018. Evolución de la inversión en América Latina y el Caribe: Hechos estilizados, determinantes y desafíos de política. Recuperado de https://www.cepal.org/es/ publicaciones/43964-estudio-economico-america-latina-caribe-2018-evolucion-la-inversion-america

Financial Times Stock Exchange. (26 de setiembre de 2019). FTSE Equity Country Classification. September 2019. Annual Announcement. Recuperado de https:// www.ftserussell.com/equity-country-classification

Fondo Monetario Internacional. (2019). World Economic Outlook, October 2019. Global Manufacturing Downturn, Rising Trade Barriers. Recuperado de https://www. imf.org/en/Publications/WEO/Issues/2019/10/01/ world-economic-outlook-october-2019
Instituto Nacional de Estadística e Informática. (2020). Panorama de la economía peruana 1950-2019. Recuperado de https://www.inei.gob.pe/biblioteca-virtual/ publicaciones-digitales/

Jurado, I., \& Ramos, C. (2021). Efectos de los shocks externos en el desempeño macroeconómico del Perú 2003 2018 (Tesis para optar el grado de título profesional de economista). Recuperada de https://repositorio.continental.edu.pe/handle/20.500.12394/8799

Nolazco, J., Legua-Lafosse, P., \& Céspedes, N. (2016). Contribución de los choques externos en el Crecimiento Económico del Perú: Un modelo semi-estructural. (DT. $\mathrm{N}^{\circ}$ 2016-006). Recuperado del sitio de Internet del Banco Central de Reserva del Perú: https://www.bcrp.gob.pe/ publicaciones/documentos-de-trabajo/dt-2016-06.html

Peña, L. (2017). Influencia del riesgo país peruano sobre la inversión extranjera directa para el periodo 2002-2016 (Tesis para optar el grado de título profesional de economista). Recuperada de https://repositorio.ulima. edu.pe/handle/20.500.12724/5213

Peñaranda, C. (2016). Disminuye la inversión extranjera directa en países de la Alianza del Pacífico. Recuperado del sitio de Internet de la Cámara de Comercio de Lima: https://apps.camaralima.org.pe/repositorioaps/0/0/par/r766_2/informeeconomico_768.pdf

Quispe, Z., Rodríguez, D., Toma, H., \& Vásquez, C. (2017). Choques externos y coordinación de políticas monetarias y macroprudenciales en las economías de la Alianza del Pacífico. Revista Estudios Económicos, (34), 31-53. Recuperado de https://www.bcrp.gob.pe/docs/ Publicaciones/Revista-Estudios-Economicos/34/ ree-34-quispe-rodriguez-toma-vasquez.pdf

Rodríguez, X., \& Díaz, A. (2020). Impacto en américa latina de caídas del crecimiento en China y Estados Unidos. Revista Académica Institucional, (3), 1-31. Recuperado de https://www.usanmarcos.ac.cr/content/ impacto-en-america-latina-de-caidas-del-crecimiento-en-china-y-estados-unidos

Ticona, J. (2019). La Bolsa de Valores de Lima y su rentabilidad respecto a las principales bolsas del mundo y la economía peruana 2009-2016 (Tesis para optar el título profesional de ingeniero económico). Recuperada de http://tesis.unap.edu.pe/handle/UNAP/10530 